\title{
Attribute Based Collusion Resistant Controlled Secure Data Processing System Using Node Insertion Method
}

Dhanalakshmi P. ( $\sim$ pdhalakshmi3@gmail.com )

Sree Vidyanikethan Engineering College

\section{Rajarajeswari S}

Vellore Institute of Technology: VIT University

Nripendra Narayan Das

Manipal University: Manipal Academy of Higher Education

Karthikeyan C

Koneru Lakshmaiah Education Foundation

\section{Chandra Sekhar Reddy}

CMR College of Engineering and Technology

\section{Parita Jain}

KIET Group of Institutions: Krishna Institute of Engineering \&amp; Technology

\section{Research Article}

Keywords: Cloud computing, attribute-based encryption, collusion resistant, data sharing, security, node insertion

Posted Date: April 21st, 2021

DOl: https://doi.org/10.21203/rs.3.rs-432580/v1

License: (9) This work is licensed under a Creative Commons Attribution 4.0 International License. Read Full License 


\title{
ATTRIBUTE BASED COLLUSION RESISTANT CONTROLLED SECURE DATA PROCESSING SYSTEM USING NODE INSERTION METHOD
}

${ }^{1}$ Dr P.Dhanalakshmi, ${ }^{2}$ Rajarajeswari S, ${ }^{3}$ Dr Nripendra Narayan Das, ${ }^{4}$ Karthikeyan C,

${ }^{5}$ L. Chandra Sekhar Reddy, ${ }^{6}$ Dr Parita Jain

${ }^{1}$ Associate Professor, Department of Computer science and Systems Engineering, Sree Vidyanikethan Engineering College, A. Rangampet, pdhalakshmi3@gmail.com

${ }^{2}$ Associate Professor, School of Computer Science and Engineering, Vellore Institute of technology, Chennai Campus, rajarajeswari.s@vit.ac.in

${ }^{3}$ Associate Professor, Department of Information Technology, Manipal University Jaipur, Rajasthan, nripendradas@gmail.com

${ }^{4}$ Associate Professor, Department of Computer Science and Engineering, Koneru Lakshmaiah Education Foundation, Vaddeswaram, Guntur, Andhra Pradesh, India. ckarthik2k@gmail.com

${ }^{5}$ Assistant Professor, Department of CSE, CMR College of Engineering \& Technology, Kandlakoya (V),Medchal-501401, chandunani@cmrcet.org

${ }^{6}$ Assistant Professor (Research),Department of Computer Science and Engineering, KIET Group of Institutions,Delhi-Meerut Road, Muradnagar, Ghaziabad, Uttar Pradesh, India, paritajain23@gmail.com

\begin{abstract}
:
In the fast-developing technological world, a large number of informational data is obtained out of different software applications and hardware sources. Data storing, sharing of data, and then processing is more effective through the cloud computing method. In practice, the issue faced is a lack of security where the user details are leaked due to the exposure of software and hardware conflict. Among different data sharing technique, cloud computing is the most convenient method to be adapted due to service satisfying features and economy. Attribute-Based-Encryption (ABE) has the potential to detect even a narrow-drawn data sharing. The drawback of the conventional attribute-based encryption (ABE) poor data security and high over-head computation. The customer side satisfaction is not achieved because of security lacking in narrow-drawn informational data sharing as well as processing. The difficulty in achieving a standardized form of data sharing is an unsolved challenge for secured data processing. The attribute-based data sharing method is more effective for secured data processing in cloud computing. Customer satisfaction can be achieved through the secured services of cloud. The customer's data should be secured and also the customer's data is under an authorized access. The protection of data in cloud computing includes the usage of hardware solutions for a secured data operation, provides support in data storage, access, sharing, and automated detecting of risk during data processing.
\end{abstract}

\section{Keywords}


Cloud computing, attribute-based encryption, collusion resistant, data sharing, security, node insertion.

\section{Introduction:}

In recent times, cloud computing is a broad range of adaptable methods in data sharing. Cloud computing can transfer data from different regions and also support long-distance communication. Data sharing is possible simultaneously among different end-user. But still, the issue faced during the data sharing and processing is the leakage of informational data of a user is the main problem in the cloud computing technique in a wide range of communication (M. Li et al., 2013; S. Wang et al., 2016). The user can control and access their data in a secure manner is the essential feature in cloud computation. Unauthorized access is a disadvantage that may reduce the usage of users and affect organizational growth. Distinct access is allowed for different users. Thus, a secured data processing system has to be developed for better data access that the informational data available comes under authorized control (S. Wang et al., 2016).

Attribute-based encryption enables us flexible access of narrow-drawn data. Attribute-based encryption is capable of encrypted sharing of data from an individual to many numbers. The access is possible with the appearance of a ciphertext and a secret key. In the data sharing process, as per the private key, the ciphertext is being decrypted considering the similarities of attribute-based access policies. The organization is responsible for setting up authorized access and securely controlling the informational data. They are even able to decide which set of users can access and maintain their data where these data are encrypted using the attribute-based encryption along with the access policies prior to conserving thecustomer's data in the server. Thus, the authorized list of owners able to share the conserved data out of cloud server (V. Goyal et al., 2006; J. Bethencourt et al., 2007).

Cloud computing involves the storing of obtained user's data in the cloud server. The public, private, and hybrid cloud are present in the cloud servers. The public cloud is not highly secured for the customer's data. In private clouding, the user's data is partially secured whereas hybrid cloud is an association of private and public clouds. The user involves in access control in a privacy manner and data deduplication when the data in the public cloud is not secured and highly sensitive (Huang et al., 2017; Li et al., 2014, 2015, 2017). The conventional accession control technique includes the user's data in the cloud server has authorized accession control only by the user and the organization. The accession control of the owner and the organization is distinct in cloud computing and hence, the cloud server provide a high security for the conserved data of the respective owners. Prevention of data is achievable only when the customer encrypts and transmits the data to ciphertext.

Attribute-based encryption (ABE) in association with cloud computing is a more apt method involved in the recognition of fine-drawn access control of stored data. The users can attain a secret key for data access in accordance with related attribute in attribute-based encryption. In an accessing method, attribute-based encryption (ABE) is divided into key-policy attribute-based encryption and ciphertext policy attribute-based encryption. In key-policy ABE, an issue facing during the access 
control by the user is confined with the private keys. Ciphertext policy includes ciphertexts both in insertion and bound (Green et al., 2011; Zhou et al., 2012).

Attribute-based encryption is also known to be fuzzy identity-based encryption (Sahai et al., 2005). In biometric encryption applications, attribute-based encryption is preferred. The algorithm is executed to determine the noise using biometric measurement at different time-periods. Error tolerance in fuzzy identity-based encryption has an ability to overcome the above-mentioned problem. The fuzzy identity-based encryption includes deformed secret key to decrypt the ciphertext to identify biometric (Goyal et al, 2006). Secure data processing involves the usage of attributebased encryption in accessing the system. Attribute-based secure data system schemes are more efficient (Chase, 2007). To prevent the data from attacks and to have a safe process attribute-based encryption is preferred ( $\mathrm{Li}$ et al., 2009). The attribute-based encryption (ABE) improves decryptionefficiency (Zhang et al., 2013, 2017).

The unbounded attribute-based encryption (ABE) is associated with large attributes and ABE is independent of attribute size for the encryption process (Lewko Waters, 2011). To a further development in attribute-based encryption is more effective in many applications (Rouselakis and Waters, 2013). To retrieve attribute-based encryption (ABE) is being attained either through direct attribute or user revocation scheme (Zhang et al, 2014). The ciphertexts are being recovered using an auxiliary function are updated. A full secured attribute-based encryption (ABE) is preferred in regards to security purposes (Waters, 2009). Cloud computing is better by utilizing ABE which includes the computation cost and sustainable size (Zhang et al, 2014) and this attribute-based encryption in association with cloud computing provide a secured data processing. (Zhang et al, 2016).

Node insertion method is implemented to have a preserved data analysis process. The node insertion method is considered as a building block of many data analysis techniques. This method combines the suitable techniques to provide a secure data processing system where the essential features of the data analysis technique are mainly recognized. Homomorphic encryption provides security to the outsourced data but still, the collusion happens when the customers count increases (Florian Kerschbaum, 2012).

Cloud storage is involved in securely storing the customer's data by utilizing the internet. Cloud computing is more suitable for storing data and accessing process in many organization as its features such as more space, safe to handle, able to share the user data by themselves through internet facility, even enable us to retrieve the data when necessary and low expensive to implement (Jingli Ren, 2016). Cloud computing is involved in storing data from a different point of view and they are public, private, personal, hybrid, and computing cloud storage. The public storage in cloud is oriented with business aspirant view where the organizational data is being outsourced to the cloud storage providers. Public cloud storage is authorized to access which is monitored under the control of the organization and this is an apt storage method for low and medium enterprises as it is low cost and provides more space (Pan Yang, 2016). A grouping of public cloud is a personal cloud storage 
method that is familiarly known as a mobile cloud but it is different from public storage and personal storage in cloud allows an individual in accessing the public stored data. The managing of stored data with the support of professional staff is known to be a private cloud. This clouding method provides high security and hence the cost to implement in private cloud storage is high. Private clouding is more apt for large level enterprises and involved in securing vulnerable data. The hybrid cloud storage method is an association of both private and public clouding. In this storage method, the customers can store important data in the private sector and other informational data of the customers in public. The specified information data of the organization can be exposed in the community through the community clouding (Jingli Ren, 2016).

Cloud computing undergoes three broad ways in storing the data.Block, file, and object storage are the different storing methods. The block storage in cloud is recognized in Storage Area Network (SAN) where the management of data is possible through the simplified interface services of web. File storage is combined with a Network Attached Storage (NAS) technology. The proper controlling and accessing of data being stored is more easy and convenient with the file storage system in comparison with the block storage system. The organization is also responsible for controlling the massive number of data which is a very challenging task to provide security and to share the data as per requirement (Neal N. Xiong, 2016).

Cloud computation includes features such as scalability, measuring resources, and accessible interfaces. Cloud storage is classified into four stages. The first stage is comprised of device storage and management. Followed by the initial stage, the primary management layer is available is considered to be the core portion of the cloud storage. The interface layer is considered to be the most resilient portion of cloud storage (Neal N. Xiong, 2016). The final layer is the access layer and thus the cloud storage includes the storing, computing, recognizing, accessing, and controlling of data. Data confidential is mainly to secure the user's informational data from unauthorized access. This process involves only authorized access and control. Data cannot be altered. The data can be accessed at any time in different places by the users through data availability. Even fine-drawn data can be accessed if possible and provide a secure sharing of informational data of the customers in the dynamic group. It also enables a resistance against the leakage of stored data. Privacy protection is provided which gives a guarantee for the stored data and customers are only able to access personal details such as location, identity, etc. The organization is responsible for maintaining the data in the cloud without any interception (Pan Yang, 2016).

\section{CONTRIBUTIONS:}

An attribute-based secure data sharing and processing is more suitable for internet based customers. Most of the tasks involved in the cloud computation process can be eliminated by making use of an attribute-based encryption (ABE) scheme in addition to system public parameters. The computational cost of ciphertext is reduced due to illegitimate ciphertext. The decryption phase is

followed by the public ciphertext phase. The customers examine the cost of the given ciphertext in 
public ciphertext phase. Data security is the main issue during the collision of a massive number of customers together. The hostile malicious attack causes a security issue that can be overcome through the node insertion method. A big data storage system is being developed for security purposes. The bid data storage system scheme will secure the data even if partial data is being leaked. The hidden data can be accessed through a policy of anonymous attribute-based broadcast encryption. The organization can send message or ciphertext to the customers where the users are enclosed in a predefined receiver group. The organizational relationship with the customers can be maintained and fulfilled through this policy. The proposed standardized system model is $\mathrm{A} 2 \mathrm{~B} 2 \mathrm{E}$ (where A2B2E expansion is anonymous attribute-based broadcast encryption) and this system model is proclaimed as it provides high security in a standardized form. A2B2E is recognized and helps in constructing a data-sharing protocol. This system is adapted by a large scale user where the users can communicate from a distinct group. An individual user has a specified group and hence the details within that group can be accessed only by a data owner. The customers are enabled to share the data simultaneously with an access policy scheme. The anonymous attribute-based broadcast is the aptest method to be carried out for a lightweight device as the computation cost will be more as per the weight of the devices and the data sharing is well-suited for the lightweight devices. In the case of light-weight devices, both the user and the sender cost of the computation process is performed in an offline mode or authorized with the cloud server. The enhanced data sharing protocol is analyzed with more simulation models and its performance shows that it is useful for contemporaneous applications.

\section{OVERVIEW:}

The attribute-based data sharing system is enhanced with the support of cloud computing in association with internet, artificial intelligence, node insertion, and collusion resistant. The user's data is secured through the cloud computing process where the data is stored and shared by the user to the multi-receivers.

\section{BACKGROUND:}

Attribute-based encryption was applicable for biometric encryption (Goyal et al, 2006) was familiarly known to be fuzzy identity-based encryption (Sahai and Waters, 2005). Fingerprint type biometric encryption was provided with various fingerprint for each time depending on the biometric calculation of noise during the extraction algorithm. The biometric encryption was highly oriented with a contemporaneous application. The fuzzy identity-based encryption was deployed with an error-tolerance can overcome the consequence that arises during the data sharing and accessing. The classic model of biometric utilizes the private key was different from the original one could provide a decrypt cipher was responsible for identification. The attribute-based encryption conceptualization was defined through a set of attributes. Attribute-based encryption was classified into two different concepts and they were KP-ABE and CP-ABE (Goyal et al, 2006). The key generation and ciphertext generation could handle error-tolerance. The access could be enabled in KP-ABE by utilizing private keys in the divided form to secure data (Goyal et al, 2006). A generic tree model access was 
supporting a $\mathrm{CP}-\mathrm{ABE}$ structure to provide a secured data processing (Benthencourt et al, 2007). In $\mathrm{CP}-\mathrm{ABE}$, the ciphertext was divided into a large count acknowledging to access policy.

The contemporaneous applications mostly make use of attribute-based encryption. Efficiency of the attribute-based encryption was recorded to represent the standardized security through this system model. The concept of ABE with Multi-authorities could reduce the conviction of attribute authority (Chase, 2007). The main reason for the enhancement of attribute-based encryption was to prevent attack of private keys (Li et al, 2009). Anonymous attribute-based encryption developed a decrypt technique in association with it to improve the efficiency of decryption (Zhang et al, 2013, 2017). A large count of attributes was available in unbounded attribute-based encryption (ABE) and the size was not limited for the attribute sets in an encryption process (Lewko and Waters, 2011). To satisfy the need of the customer and secure the data of the users, an attribute-based encryption scheme was approached universally (Rouselakis and Waters, 2013). The user revocation methodology was preferred for uninterrupted access was employed for flexible ABE (Zhang et al, 2014) in which the ciphertext was involved in the revocation methodology. The ciphertext included in revocation was being rehabilitated. A highly secured $\mathrm{ABE}$ was enhanced to provide user data security (Waters, 2009). The computation cost and the space occupation of the ciphertext were the characteristics considered for cloud computing utilizing ABE (Zhang et al, 2014) and mobile cloud computing enabled data sharing through the realization of attributes (Zhang et al, 2016). Analysis of access complexity could be measured in considering the features such as computational cost in attribute-based encryption (ABE) in an offline or online mode (Hohenberger and Waters, 2014).

Cloud computing was involved in access control to enable security to fine-drawn data with the support of PHP data and attribute-based encryption (ABE) properties. Some of the attribute-based encryption $(\mathrm{ABE})$ were attribute-based broadcast encryption (ABBE) and privacy-maintenance attribute-based encryption. Accession control mechanism along with attribute-based encryption enables a user to share the data among different users through the leveraging multi-authority attribute-based encryption (MA-ABE) ( $\mathrm{Li}$ et al, 2013). In case of necessity, the user could make use of the revocation function to privileges the access. Multi-authority attribute-based encryption (MA$\mathrm{ABE}$ ) scheme was responsible in respect to identifyand securing user data and this scheme enabled to overcome the issues during the data sharing (Zhou et al, 2015) and the revocation process as consisting of attributes, revocation storage, and controlled access to provide the privacy security and support in sharing data to multilevel where the data was stored through cloud computing. Attributebased encryption might allow the authority to access and control data side by side with the anonymous and guaranteed legal sharing of data (Liu et al, 2015). The attribute-based broadcast encryption (ABBE) could have recognized access and control over fine-drawn data and this encryption enabled a multifunctional security framework to protect the user's data (Narayan et al, 2010). This attribute scheme was mainly employed to maintain the user's privacy to access the stored data through cloud computing and to sustain the data confidentially.

Partially hidden access control was associated with attribute-based encryption (ABE) to ensure privacy of user (Nishide et al, 2008). The private details of the user should be kept confidentially 
whereas the name of the user can be spotted by anyone. The enhancement of the conjunctive access policy technique provides access control in a novel approach (Zhou et al, 2015). The user's hidden data were disclosed due to the wildcard attribute-based access and the privacy of the user was not achievable (Zhou et al, 2015). The vector-based access policy was utilized for the employment of a highly-secured $\mathrm{CP}-\mathrm{ABE}$ scheme. This scheme includes the user's attributes to apply the product using encryption techniques and hence the hidden data privacy was being analyzed. Depending on the number of attributes, ciphertext size and computational cost can be determined and it is uneconomical in the case of light-weight devices. The data-sharing system was associated with the PHR data measurement for a dynamic process (Phuong et al, 2016).

Broadcast encryption (BE) permitted the broadcaster to transmit the data from an individual to multigeographical customers through an insecure channel. BE was the same as that of ABE where communication occurs between single to many customers. In $\mathrm{BE}$, the data shared by the user was undergoing an encryption process whereas the receivers could perform a decryption process. The user should have the potential to manage the multiple receivers as an individual in broadcast encryption. Sometimes it was challenging to obtain the data of the multiple receivers in large-scale communication (Naor et al, 1993). Association of ABE and BE was familiarly known as Attributebased broadcast encryption (ABBE). Direct revocation could be achieved through attribute-based encryption $(\mathrm{ABE})$. The sender rejected the revoked user from the receiver group. To have an effective revocation directly, ABBE should possess the receiver list. The sender was able to communicate with many encryptions with broadcast support. The ciphertext-policy ABBE (CPABBE) will permit the broadcaster or encryption dynamically to promote the subgroups of users with a specified attribute (Lubicz et al, 2008). The development of ciphertext-policy and key-policy environments included the association of both BE (Boneh et al, 2005) and ABE (Waters et al, 2011) to form an ABBE scheme (Attrapadung et al, 2009). The logical gates such as AND, OR, and NOT gates were utilized for access control in an ABBE scheme. The scheme with gates size increases linearly concerning complex access formula (Junod et al, 2010). The proposed attribute-based broadcast encryption (ABBE) could achieve steady size of ciphertexts and this scheme was economically low cost (Zhou et al, 2010). The proposed ABBE was classified into KP-ABBE and $\mathrm{CP}-\mathrm{ABBE}$ scheme was responsible for privacy checking (Phuong et al, 2015).

\section{SYSTEM MODEL:}

Attribute-based encryption (ABE) is recognized to produce centralized data sharing among a large count of users is shown in Figure1 and Figure2. The customer or user from the different association or share enables to transfer data to cloud and the customers can share their data to the receiver of other sector or organization. The features which come under the system model are Trusted Authority (TA), Cloud Storage Server (CSS), Decryption-Cloud Service Provider (D-CSP), the data owner, and the organizational potential to share data to a large number of receivers. The framework of the system is designed with the Trusted Authority and depending on the set of attributes and specified group, the user's secret key. The Cloud Storage Server is prominent to protect the data being stored by the user in the server and this secures the data confidentially to the best. CSS includes protocol 
identification and the retrieving of ciphertext data for information gathering. The customers can communicate with the receiver through the communication devices such as mobile phones and the sender and the receiver of the same organization can contact each other. The decryption task can be achieved through Decryption Cloud Service Provider (D-CSP) and this service provider undergoes outsourcing computation produces an inaccurate result that is being kept analyzed by the user. The data-sharing system permits ciphertext access by the user and user's identity is being determined in the confined list. The users can access the policy where the desired attribute of a particular user is available. The important feature in the designed protocol can access the ciphertext as per the access policy independent of the leakage of information of the users.

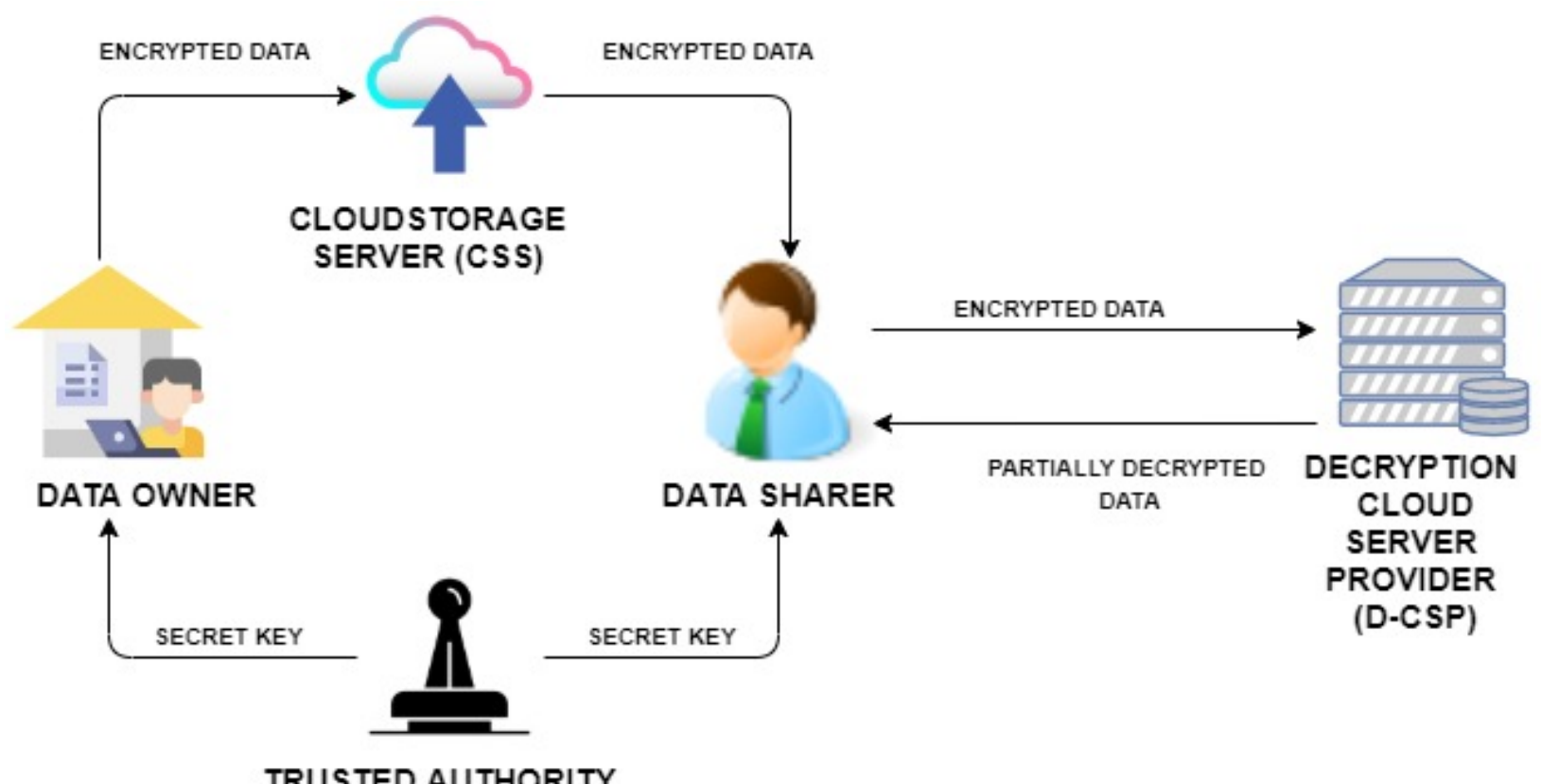

(TA)

Figure 1:Data Sharing.

The sender can share the data with multiple users who are belonging to different organizations based on the group habituated data sharing system through the cloud server. The user can be monitor and control their data and able to transfer the data to the receiver concerning the group identities according to the desired access policy. Ciphertext was speeded up by an online or offline encryption process where the users undergo a heavy computation process that occurs in an offline mode and there will only mild process in an online computation. Authorized accession is permitted for others apart from user where the private key is transformed to the Decryption Cloud Service Provider (DCSP). D-CSP is recognized for sharing the informational data partially to the receivers. The outsourced ciphertext is checked and the retrieval key is attained by the receivers. 


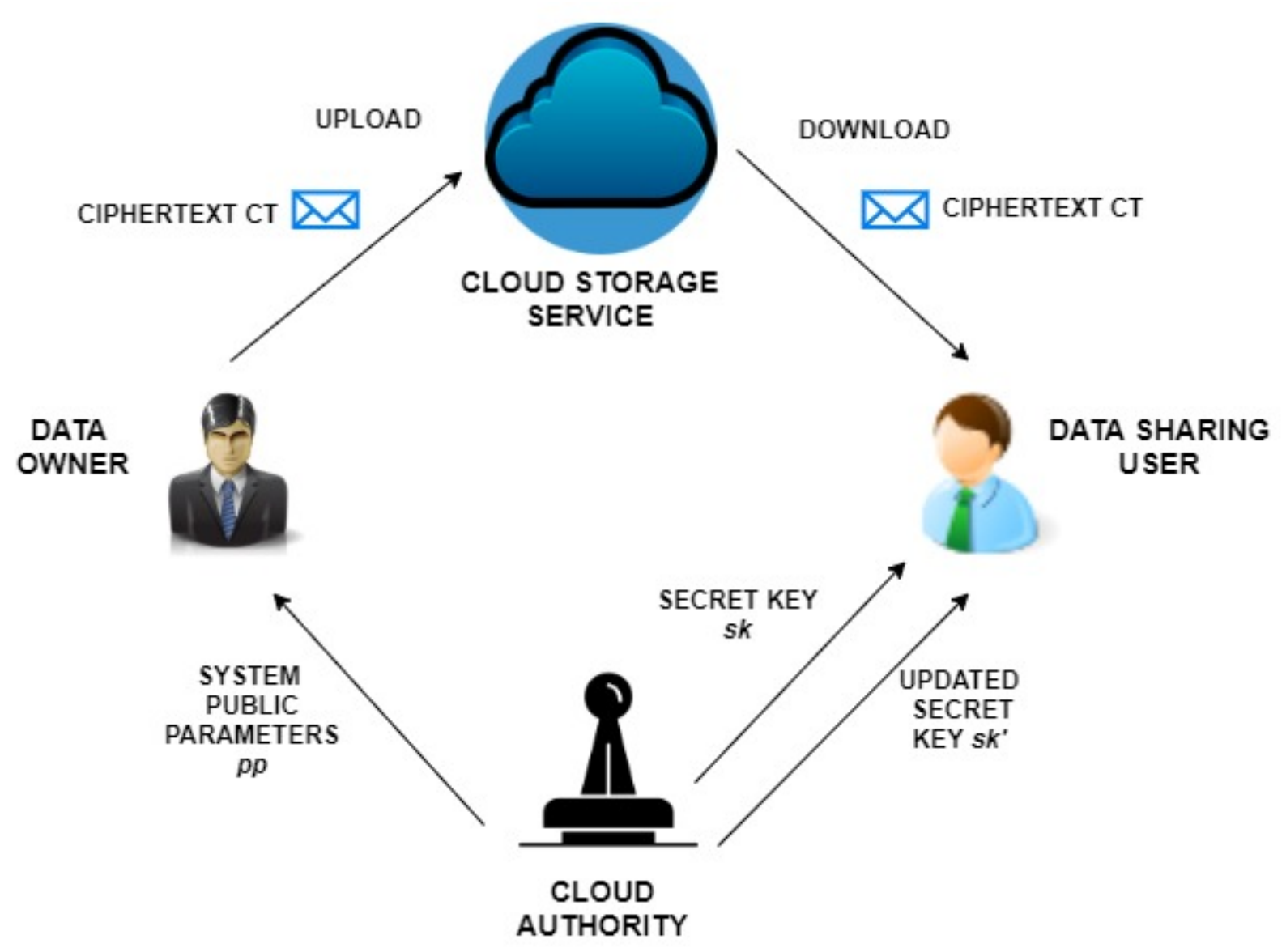

Figure 2: Attribute based encryption (ABE).

\section{OBJECTIVES:}

The main aim of the enhanced protocol-oriented data sharing systematic model is to share data among different groups or organizations under the privacy mode in the service server of cloud where data are stored during the computation process.

The data shared by the user to the multi receivers can be accessed through an unauthorized identity that will be notified to the user with the support of the attribute depending on access policy and the unauthorized access is also due to the insufficient attribute. The main issue faced during the data processing is the collusion attacks which is unable to retrieve ciphertext, the association of attributes. The individual from the multi-receiver cannot decrypt the desired ciphertext.

The access policy is highly associated with ciphertext for the security purpose and the stored data of an individual has to be maintained confidentially even from the legitimate data receivers. The responsibility of the ciphertext is to block the sensitive informational data transfer of an individual user where the data is conserved in the cloud server. 
Data processing efficiency is improved in two methods. The first way, where the users able to carry a heavy computation through communication devices such as mobile phones independent of the encryption of data in an offline mode. A partial process of encryption is carried out in an online mode. The protocol analyzed a large number of outsourced computations to Decryption Cloud Service Provider (D-CSP). The enhanced protocol allows data sharer to access local computation which is quite less than the decryption process.

\section{ARCHITECTURE:}

The enhanced attribute-based data sharing (ABDS) system model is an appropriate method for a constrained user in cloud computing. This attribute-based data sharing system includes features such as Attribute Authority (AA), Mobile Data Owner (MO), Data User (DU), and Cloud Service Provider (CSP).

Attribute in public phase and master key of the system are attained through an Attribute Authority (AA). The ciphertext present in the system under the public specification is acquired by a Mobile Data Owner (MO) in an online phase. The characteristics features of attribute-based data sharing are highly trusted and hence, the Attribute Authority can manipulate the users.

The Mobile Data Owner (MO) enables the data stored in cloud storage service to secure and maintain their data more confidentially which is being monitored and controlled by Control Service Provider (CSP). Data sharing is performed by a user as the data are maintained by CSP. During the power access, Mobile Data Owner has permission to send ciphertext even in an offline phase but still, the messages are received by the receiver during the online phase will not affect the battery life.

The ciphertext data generated by Mobile Data Owner (MO) is being stored and controlled by the Cloud Service Provider (CSP). CSP includes a large count of servers to permit data storing where the cloud server is emphasized by a manager of a data service.

The ciphertext stored in the Cloud Service Provider (CSP) is accessed using a secret key by a Data User (DU). The performance of the public ciphertext phase followed by the decryption phase is responsible for improving the efficiency of the decryption. Data User downloads the ciphertext stored in the CSP where the ciphertext has to pass the public test phase followed by decryption phase. 


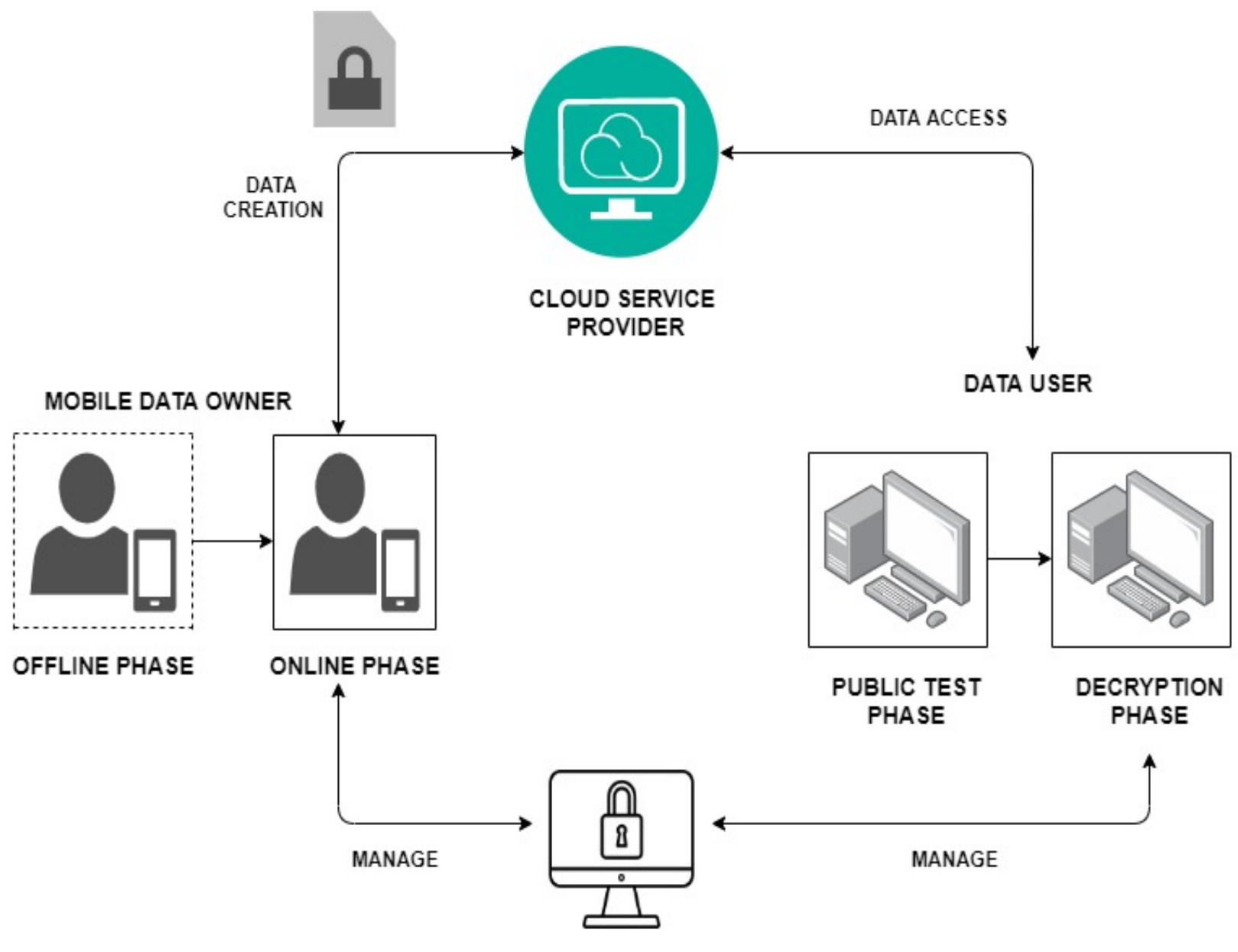

ATTRIBUTE AUTHORITY

Figure 3: Attribute-based data sharing (ABDS) system model.

In attribute-based broadcast encryption (ABBE), user secret key SK is produced depending on their identity ID and attribute $U$. The user identity set $\mathrm{S}_{\mathrm{ID}}$ is linked with the decryption ciphertext and access policy performance is based on the SK.

$\mathrm{U} \mid=\mathrm{AS}$ and $\mathrm{ID} \in \mathrm{S}_{\mathrm{ID}}$

In anonymous attribute-based broadcast encryption $\left(\mathrm{A}^{2} \mathrm{~B}^{2} \mathrm{E}\right)$, ciphertext encloses the access policy W. The algorithm for $\left(A^{2} B^{2} E\right)$ scheme is given below,

$\operatorname{Setup}\left(1^{\lambda}\right)$ : The output of the algorithm consists of public parameter of a system PP and master secret keys MS with the assistance of security parameter $\lambda$.

Encrypt ( $\left.\mathrm{S}_{\mathrm{ID}}, \mathrm{AS}, \mathrm{m}, \mathrm{PP}\right)$ : Algorithm is performed respectively by data owner for the purpose of ciphertext (CT) generation depending on user identity set $\mathrm{S}_{\mathrm{ID}}$, access structure AS, message $(\mathrm{m})$ and PP. 
KenGen (ID, U, PP, MS): Depending on the user's identity ID and the attribute set U, PP and MS, this algorithm produces the secret key SK as output.

Decrypt (PP, CT, SK): The output of this algorithm is message $(\mathrm{m})$ based on PP, CT and SK.

Anonymous attribute-based broadcasting encryption $\left(\mathrm{A}^{2} \mathrm{~B}^{2} \mathrm{E}\right)$ provide the security to the IND-CPA for the selected users in the following way:

The adversary ' $A$ ' performs influentially to an attribute set $\mathrm{S}$ * and transmit it to challenger B. ' $A$ ' high secured parameter $\lambda$ is selected by the challenger B followed by this the execution of Setup algorithm carried out to obtain the master key (MK) and the public key (PK). In this algorithm, Conservation of MK and produces the PK to ' $\mathrm{A}$ '. In phase1, the key generation oracle and decryption oracle are linked accompanied by adversary ' $A$ ' queries. In the key generation oracle, $S *$ is unable to accept the access structure sent by the adversary ' $A$ '. For accessing the structure, the challenger $B$ produces the secret key to ' $A$ '. In decryption oracle, adversary ' $A$ ' relinquish a ciphertext $\mathrm{CT}_{\mathrm{S}}$ message $m$ concerning to an attribute set $\mathrm{S}$. If the ciphertext $C T_{S}$ is legitimate then the message $m$ is sent back by the challenger B. After completing these process, comparison of any two message length $\mathrm{m}_{\mathrm{o}}$ and $\mathrm{m}_{1}$ is challenging concerning $\mathrm{S}^{*}$. The challenger $\mathrm{B}$ throws a random coin $\mathrm{b}$ in a range between 0 and 1 . Computation of ciphertext with the attribute set and sends the output of the computation process to adversary 'A'.

$\mathrm{B} \in\{0,1\} \quad(1)$

$\mathrm{CT}_{\mathrm{S}^{*}}=\operatorname{Encrypt}_{\text {on }}\left(\mathrm{PK}, \mathrm{m}_{\mathrm{b}}, \mathrm{S}^{*}, \mathrm{CT}_{\text {off }}\right)$

Where $\mathrm{CT}_{\text {off }}=$ Encrypt $_{\text {off }}(\mathrm{PK})$

Similarly, phase 2 also not accepts the $\mathrm{CT}_{\mathrm{S}^{*}}$ provided by the decryption oracle. The adversary 'A' produces the guess bit b' ranging in between 0 and 1 and it comes to the conclusion only if $c^{\prime}$ ' $=\mathrm{c}$. The adversary 'A' produces security key in attacking the KP-ABE is given as follows,

$\operatorname{Adv}^{\mathrm{KP}-\mathrm{ABE}},{ }_{\mathrm{A}} \cdot(\lambda)=\mid \operatorname{Pr}\left[\mathrm{c}^{\prime}=\mathrm{c}\right]-1 \frac{1}{2}(3)$

Universal attribute in system is denoted as

$\mathrm{A}=\left\{\mathrm{A}_{1}, \mathrm{~A}_{2}, \ldots \ldots \ldots, \mathrm{A}_{\mathrm{L}}\right\}$

$A_{i}(i \in\{1, \ldots, L\})$

where $A_{i}$ is the association of both positive and negative values. The group is allotted for each user GID and these user comes under the attribute list. Attribute list, a group identity set and an ANDgate access policyare denoted as

$\mathrm{U}=\left\{\mathrm{U}_{1}, \ldots \ldots ., \mathrm{U}_{\mathrm{L}}\right\}$

$\mathrm{S}_{\mathrm{GID}}=\left\{\mathrm{GID}_{1}, \ldots \ldots \ldots, \mathrm{GID}_{\mathrm{m}}\right\}$

$\mathrm{W}=\left\{\mathrm{W}_{1}, \ldots \ldots \ldots, \mathrm{W}_{\mathrm{L}}\right\}$ 
Apart from the Attribute Authority, other features are considered to be authoritative. The features are assigned to perform the task being allotted and there is a chance of informational private data leakage. The main aim for the development of an attribute-based data sharing (ABDS) system model includes confidential maintenance of data and Collusion-Resistant. The ciphertext of the customer is not access permitted by an unauthorized user and also includes the accessing permission by the Cloud Service Provider (CSP) to ciphertext message. Action of transferring data to the cloud server by multiple users simultaneously will result in the collision and hence, the decryption process is not succeeding.

From Figure3 we infer that the mobile constrained user utilizes this data-sharing system where the message sent is very quickly converted to an attribute-based encrypt ciphertext. The mobile user makes use of all attributes in accessing the power supply. An individual can cross-check whether the ciphertext is legitimate without the dependence of a secret key. The counterfactual ciphertext is detected in the decryption phase and hence, these ciphertexts are removed from the process. There are five phases involved in the developed attribute data sharing system for a limited user. They are initialism, customer enrollment, data formation in an offline phase, data formation in an online phase, and accession of data.

Initially, the Attribute-Authority (AA) includes public attributes and master keys. The attribute of the system public sector is utilized by the users, where the measurement of ciphertext is analyzed through AA. The Mobile Data Owner (MO) has permission to use the public attribute in an online phase. The customer is asked to couple with an attribute-based data sharing method by accessing the Attribute Authority (AA) during the enrollment of customers. Depending on the accessing methodology, the user is provided with a secret key to maintain their details confidentially. The Mobile Data Owner (MO) can send the message during the offline phase but still, the accessing of a message is encountered during the online phase. The MO proceeds with the data sharing during the

online phase where the attribute set is withdrawn from Cloud Service Provider (CSP) for sharing. If ciphertext stored in the CSP is legitimate then Data Access (DU) can download the ciphertext from CSP. Data access allows the Multi-users (MU) to decrypt based on their secret keys.

\section{PERFORMANCE EVALUATION:}

The attribute-based data sharing (ABDS) system model improves the security of the user's data (Hohenberger and Waters, 2014; Lewko and Waters, 2011; Liu et al., 2014; Rouselakis and Waters, 2013). Bilinear group development includes pairing, exponential, and multiplication operations. Arithmetic operation cost is ignored in system development. Offline ciphertext's size is acquired through the determination of attribute universe's size, complexity of access structure and attributes bound with a ciphertext are considered for the performance evaluation. The public test cost, decryption cost, online encryption cost, offline encryption cost, and security all are considered and analyzed. In cloud computing, the data sharing process is carried by the constrained users will have a reduced number of computation tasks during the offline phase. The Linear Secret Sharing Schemes 
(LSSS) controls all scheme involved in this system and allow the processing of key policies. The proposed scheme mainly depends on the exponentiation operation which is linearly proportional to offline ciphertext size (Hohenberger and Waters, 2014). The decryption phase test is carried out after the public ciphertext test where the unwanted fraudulent ciphertext is detected and eliminated (Liu et al., 2014). This proposed scheme is more effective due to the public ciphertext test mechanism where the pairing operation remains constant concerning the access structure complexity. The proposed system can provide security against the Chosen-Ciphertext Attacks and Chosen-Plaintext Attacks (Liu et al., 2014). The proposed scheme is larger than the size in Hohenberger and Waters (2014), the size of the secret key is also small and is enhanced with a small ciphertext in an offline phase. The key generation phase (KP-ABE) is capable of performing in both online and offline phase simultaneously, undergoes the public ciphertext test and effectively secure the data against the Chosen-Ciphertext Attacks (CCA2). The performance evaluation of proposed scheme, implementation and computational cost are discussed deeply. The proposed system output simulation shows that the scheme is based on the Java Pairing-Based Cryptography (JPBC) (Caro and Iovino, 2011) and OS operation system inbuilt smartphone. The Java Pairing-Based Cryptography (JPBC) includes the exponentiation and multiplication operation for effective performance in both online and offline encryptions in mobile phones. The simulation result also depends on Pairing-Based Cryptography (PBC) (Lynn) and Intel Core processor machines. PBC includes exponentiation, multiplication, and pairing operation for the effective performance of public ciphertext test and decryption test to eliminate the fraudulent ciphertext by implementing in the personal computer itself. The proposed system results show that the cost of the online encryption phase, offline encryption phase, decryption phase and public ciphertext phase is costly and hence, the attribute-based data sharing (ABDS) system is preferred only the constrained users in cloud computing.

\section{RESULT AND DISCUSSION:}

In attribute based encryption (ABE), logic gate such as AND gate in addition to wildcard type scheme is used. The distinct features between the proposed system and existing system is observed with reference to size and cost for both encryption and decryption time. Secret key, ciphertext and public key size are analyzed and compared. Computational cost of the data owner is expressed as follows,

$(12 n+2) \mathrm{E}_{\mathrm{G}}+\mathrm{E}_{\mathrm{T}},(\mathrm{n}+4) \mathrm{E}_{\mathrm{G}}+\mathrm{E}_{\mathrm{T}},(3 n+1) \mathrm{E}_{\mathrm{G}},(2 n+1) \mathrm{E}_{\mathrm{G}}$.

Which provide the linear relationship with $\mathrm{n}$ number of attributes. In an encryption algorithm, $5 \mathrm{E}_{\mathrm{G}}$ is required in comparison with other schemes. The data being shared by the sharer takes a time cost of $4 \mathrm{nP}, 7 \mathrm{P}+2 \mathrm{dE}_{\mathrm{T}}, 2 \mathrm{nP}$ while the total value is $2 \mathrm{P}+2 \mathrm{E}_{\mathrm{G}}+\mathrm{E}_{\mathrm{T}}$. The proposed system provides the better performance in case of both data owner as well as data receiver side. Representation of communication cost is given as follows,

$\left.\left.\left.(4 n+2)\right|_{\mathrm{G}}\right|_{+}\right|_{\mathrm{G}_{\mathrm{T}}} \mid$ and $\left.\left.\left.\left.\left.4\right|_{\mathrm{G}}\right|_{+}\right|_{\mathrm{G}_{\mathrm{T}}}|, 2|_{\mathrm{G}}|+| \mathrm{G}_{\mathrm{T}}|,(4 \mathrm{n}+3)|_{\mathrm{G}}\right|_{+}\right|_{\mathrm{G}_{\mathrm{T}}} \mid+\mathrm{S}$. 
The experimental simulation is obtained for the theoretical analyzation of attribute based broadcast encryption (ABE) depends on pairing of java based cryptography and smartphone. This experiment is analyzed in the smartphones such as HUAWEI Honor $7 \mathrm{i}$ which has a capacity of 1.5 gigahertz processor with 3 gigabyte memory space and runs on Emotion UI. The experiment is performed in an elliptic curve formation with a value of 160-bit to obtain a security level of 80 -bit value. The finite bit count of 512 is required to build the super-singular curve. Kilobyte (KB) is the unit of communication overhead whereas millisecond (ms) is the unit of computation cost.

Table1 shows the cost of encryption time of the data owner at different system attributes. The decryption time cost of data sharer is obtained at different attributes is represented in Table2. The proposed system is capable of secure data processing from an individual to a multi-user. The communication cost in Kilobyte (KB) of ciphertext at different access structure attributes are inferred from Table 3 and Table4. The efficiency of the attribute based broadcast encryption is high.

\begin{tabular}{|c|c|c|}
\hline $\begin{array}{c}\text { NUMBER OF SYSTEM } \\
\text { ATTRIBUTES }\end{array}$ & $\begin{array}{c}\text { ENCRYPTION TIME } \\
\text { (milliseconds) }\end{array}$ & $\begin{array}{c}\text { ENCRYPTION TIME IN } \\
\text { DATA SHARING } \\
\text { PROTOCOL } \\
\text { (milliseconds) }\end{array}$ \\
\hline 5 & $0.8 \times 10^{4}$ & 0.01 \\
\hline 10 & $1.2 \times 10^{4}$ & 0.01 \\
\hline 20 & $2.8 \times 10^{4}$ & 0.01 \\
\hline 30 & $3.9 \times 10^{4}$ & 0.01 \\
\hline 45 & $5.1 \times 10^{4}$ & 0.01 \\
\hline
\end{tabular}

TABLE 1: Analyzation of encryption time cost at different attributes.

\begin{tabular}{|c|c|c|}
\hline $\begin{array}{c}\text { NUMBER OF SYSTEM } \\
\text { ATTRIBUTES }\end{array}$ & $\begin{array}{c}\text { DECRYPTION TIME } \\
\text { (milliseconds) }\end{array}$ & $\begin{array}{c}\text { DECRYPTION TIME IN } \\
\text { DATA SHARING } \\
\text { PROTOCOL (milliseconds) }\end{array}$ \\
\hline 10 & $0.8 \times 10^{4}$ & 0.02 \\
\hline 20 & $1.4 \times 10^{4}$ & 0.02 \\
\hline 30 & $2.2 \times 10^{4}$ & 0.02 \\
\hline 40 & $3 \times 10^{4}$ & 0.02 \\
\hline 50 & $3.8 \times 10^{4}$ & 0.02 \\
\hline
\end{tabular}

TABLE 2: Analyzation of decryption time cost at different attributes. 


\begin{tabular}{|c|c|c|c|}
\hline $\begin{array}{c}\text { ACCESS } \\
\text { STRUCTURE } \\
\text { ATTRIBUTES }\end{array}$ & $\begin{array}{c}\text { CIPHERTEXT } \\
\text { COMMUNICATION } \\
\text { COST(KB) } \\
\mathbf{I}=\mathbf{5}\end{array}$ & $\begin{array}{c}\text { CIPHERTEXT } \\
\text { COMMUNICATION } \\
\text { COST(KB) } \\
\mathbf{I = 1 0}\end{array}$ & $\begin{array}{c}\text { CIPHERTEXT } \\
\text { COMMUNICATION } \\
\text { COST(KB) } \\
\mathbf{I = 1 5}\end{array}$ \\
\hline 20 & 8 & 5 & 4 \\
\hline 30 & 13 & 9 & 8 \\
\hline 35 & 16 & 13 & 10 \\
\hline 40 & 19 & 15 & 13 \\
\hline 50 & 24 & 22 & 18 \\
\hline
\end{tabular}

TABLE 3: Communication cost of ciphertext compared under different attributes.

\begin{tabular}{|c|c|}
\hline ACCESS STRUCTURE ATTRIBUTES & $\begin{array}{c}\text { CIPHERTEXT COMMUNICATION } \\
\text { OVERHEAD COST(KB) } \\
\mathbf{n}=\mathbf{5 0}\end{array}$ \\
\hline 5 & 23 \\
\hline 15 & 18 \\
\hline 25 & 13 \\
\hline 35 & 9 \\
\hline 45 & 4 \\
\hline
\end{tabular}

TABLE 4: Overhead communication cost of ciphertext compared under different attributes.

\section{CONCLUSION:}

In this proposed work, security issues are deeply discussed during data sharing in cloud computing. The enhanced attribute-based data sharing (ABDS) system is more apt for limited number of data owners to enable sharing using node insertion. Resistance against collusion is reduced in this proposed where secure data processing is carried out. This system act as a barrier against leakage resilience and hence, protect the data from unauthorized access. The performance evaluation indicates that attribute-based data sharing (ABDS) practically provides high data security in cloud computing. This scheme can eliminate the fraudulent ciphertext during public ciphertext test and decryption test. The attribute set provides security to the data stored and reduce the ciphertext and pairing attacks. The simulation output of the proposed system model is more convenient for mobile users. The main goal of the protocol-oriented data sharing system enable data owner to share their data to a multi-receiver under the privacy security of the data with the resistance against collusion in cloud computing. 


\section{Declaration}

\section{Funding}

Not Applicable

\section{Conflicts of interest/Competing interests}

The authors declare that they have no conflict of interest. This article does not contain any studies with human participants or animals performed by any of the authors.

\section{Availability of data and material}

Not Applicable

\section{Code availability}

Not Applicable

\section{REFERENCE:}

M. Li et al., "Scalable and secure sharing of personal health records in cloud computing using attribute-based encryption," IEEE Trans. Parallel Distrib. Syst., vol. 24, no. 1, pp. 131-143, Jan. 2013.

S. Wang et al., "An efficient file hierarchy attribute-based encryption scheme in cloud computing," IEEE Trans. Inf. Forensics Security, vol. 11, no. 6, pp. 1265-1277, Jun. 2016.

S. Wang et al., "Attribute-based data sharing scheme revisited in cloud computing," IEEE Trans. Forensics Security, vol. 11, no. 8, pp. 1661-1673, Aug. 2016.

V. Goyal et al., "Attribute-based encryption for fine-grained access control of encrypted data," in Proc. 13th ACM Conf. Comput. Commun. Security, 2006, pp. 89-98.

J. Bethencourt, A. Sahai, and B.Waters, “Ciphertext-policy attribute-based encryption,” Proc. IEEE Symp. Security Privacy, 2007, pp. 321-334.

Huang Z, Liu S, Mao X, Chen K, Li J. Insight of the protection for data security under selective opening attacks. Inf Sci (Ny) 2017; 412-413:223-41.

Li J, Chen X, Li M, Li J, Lee PP, Lou W. Secure deduplication with efficient and reliable convergent key management. IEEE Trans Parallel Distrib Syst 2014; 25(6):1615-25.

Li J, Li J, Chen X, Jia C, Lou W. Identity-based encryption with outsourced revocation in cloud compuiting. IEEE Trans Comput 2015;64(2):425-37. Doi:10.1109/TC.2013.208. 
Li P, Li J, Huang Z, Li T, Gao C-Z, Yiu S-M, et al. Multi-key privacy-preserving deep learning in colud computing. Cluster Comput 2017; doi:10.1007/s1058-017-0849-9.

Green M, Hohenberger S, Waters B. Outsourcing the decryption of abecipertexts. In: Proceedings of the $20^{\text {th }}$ USENIX Association; 2011. P. 34.

Zhou Z, Huang D. Efficient and secure data storage operations for mobile cloud computing, in: $8^{\text {th }}$ International conference on Network and service management. IEEE 2012;37-45.

Sahai A, Waters B. Fuzzy identity-based encryption. In: Cramer R, editor. Advances in cryptology - EUROCRYPT 2005, vol. 3494. Lecture Notes in Computer Science. Springer Berlin / Heidelberg; 2005. P. 457-73.

Goyal V, Pandey O, Sahai A, Waters B. Attribute-based encryption for fine-grained access control of encrypted data. In: Proceedings of the $13^{\text {th }}$ ACM conference on computer and communications security. 2006. p. 89-98.

Bethencourt J, Sahai A, Waters B. Ciphertext-policy attribute-based encryption. In: IEEE symposium on security and privacy 2007.p. 321-34.

Chase M. Multi-authority attribute based encryption. In: Vadhan S, editor. Theory of Cryptography, vol. 4392. Lecture Notes in Computer Science. Springer Berlin / Heidelberg; 2007. p. 515-34.

Li J, Ren K, Zhu B, Wan Z. Privacy-aware attribute-based encryption with user accountability. In: Samarati P, Yung M, Martinelli F, Ardagna C, editors. Information Security, vol. 5735. Lecture Notes in Computer Science. Springer Berlin / Heidelberg; 2009. p. 347-62.

Zhang Y, Chen X, Li J, Wong DS, Li H. Anonymous attribute-based encryption supporting efficient decryption test. In: Proceedings of the $8^{\text {th }}$ ACM SIGSAC symposium on Information, computer and communication security. ACM; 2013. p. 511-16.

Zhang Y, Chen X, Li J, Wong DS, Li H, You I. Ensuring attribute privacy protection and fast decryption for outsourced data security in cloud computing. Inf Sci (Ny) 2017; 379: 42-61.

Lewko A, Waters B. Unbounded hibe and attribute-based encryption. In: Advances in cryptologyEUROCRYPT 2011. Springer; 2011. p. 547-67.

Rouselakis Y, Waters B. Practical constructions and new proof methods for large universe attributebased encryption. In: Proceedings of the 2013 ACM SIGSAC conference on computer and communications security. ACM; 2013. p. 463-74.

Zhang Y, Zheng D, Chen X, Li J, Li H. Computationally efficient ciphertext-policy attribute-based encryption with constant-size ciphertexts. In: Provable security. Springer; 2014. p. 259-73.

Waters B. Dual system encryption: realizing fully secure hibe and hibe under simple assumptions. In: Halevi S, editor. Advances in cryptology - CRYPTO 2009, vol. 5677. Lecture Notes in Computer Science. Springer Berlin / Heidelberg; 2009. p. 619-36.

Zhang Y, Zheng D, Chen X, Li J, Li H. Efficient attribute-based data sharing in mobile clouds. Pervasive Mob Comput 2016; 28:135-49. 
Hohenberger S, Waters B. Online / Offline attribute-based encryption. In: Public-key cryptographyPKC 2014. Springer; 2014. p. 293-310.

Caro AD, Iovino V. Proceedings of the $16^{\text {th }}$ IEEE symposium on computers and communications, ISCC 2011. IEEE; 2011. p. 850-5.

Lynn B. The pairing-based cryptography library. Available from: http://crypto.stanford.edu/pbc/.

Florian K. Collusion-Resistant Outsourcing of Private Set Intersection, SAC' 12 March 25-29, 2012 , copyright 2011 ACM 978-1-4503-0857-1/12/03.

T. Bhatia and A. Verma, "Data security in mobile cloud computing paradigm: A survey, taxonomy and open research issues," J. Supercomput., vol. 73, no. 6, pp. 2558-2631, Jun. 2017.

IBM. Block. Accessed on Feb. 2020. [Online]. Available: https://www.ibm.com/cloud/learn/blockstorage.

M. B. Mollah, M. A. K. Azad, and A. Vasilakos, "Security and privacy challenges in mobile cloud computing: Survey and way ahead." Journal of Network and Computer Applications, vol. 84, pp. 38-54, Apr. 2017.

Spectralogic, "Comparing File (NAS) and Block (SAN) Storage." [Online]. Available: https://edge.spectralogic.com/?\&fuseaction=home.displayFile\&DocID=4630.Accessed on: Mar. 1,2020 .

Z. Xia, N. N. Xiong, A. V. Vasilakos, and X. Sun, "EPCBIR: An efficient and privacy-preserving content-based image retrieval scheme in cloud computing," Informational sciences, vol. 387, pp. 195-204, May 2017.

Z. Xia, T. Shi, N. N. Xiong, X. Sun and B. Jeon, "A Privacy-Preserving Handwritten Signature Verification Method Using Combinational Features and Secure KNN," IEEE Access, vol. 6, pp. 46695-46705, Aug. 2018.

J. Zhang, B. Chen, Y. Zhao, X. Cheng, and F. Hu, "Data Security and Privacy-Preserving in Edge Computing Paradigm: Survey and Open Issues,” IEEE Access, vol. 6, pp. 18209-18237, Mar. 2018.

D. Zhe, W. Qinghong, S. Naizheng and Z. Yuhan, "Study on Data Security Policy Based on Cloud Storage," in 2017 IEEE $3^{\text {rd }}$ International Conference on Big Data Security on Cloud (BigDataSecurity), IEEE International Conference on High Performance and Smart Computing (HPSC), and IEEE International Conference on Intelligence Data and Security (IDS), Beijing, China, 2017, pp, 145-149. 
Figures

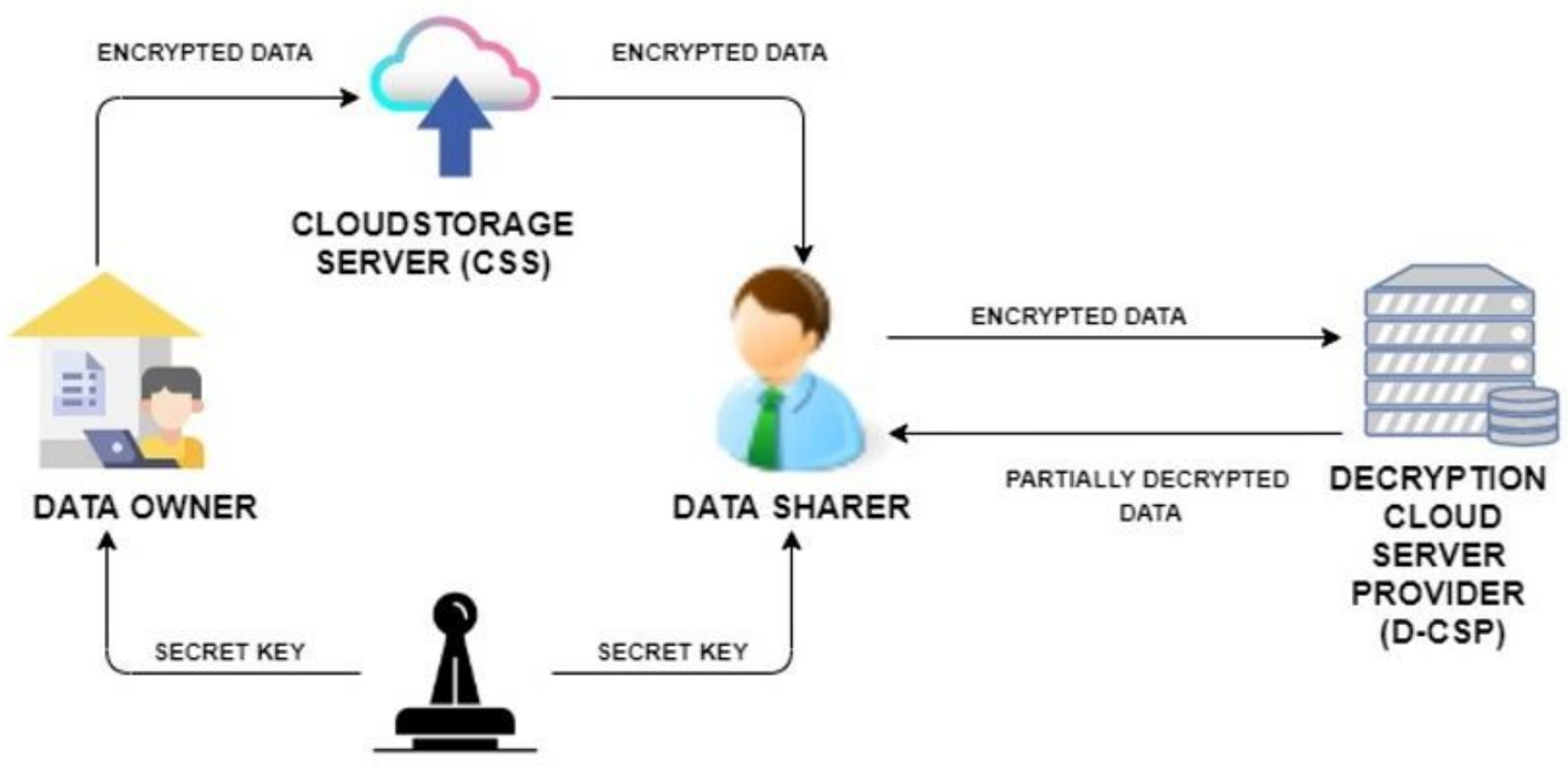

TRUSTED AUTHORITY

(TA)

Figure 1

Data Sharing. 


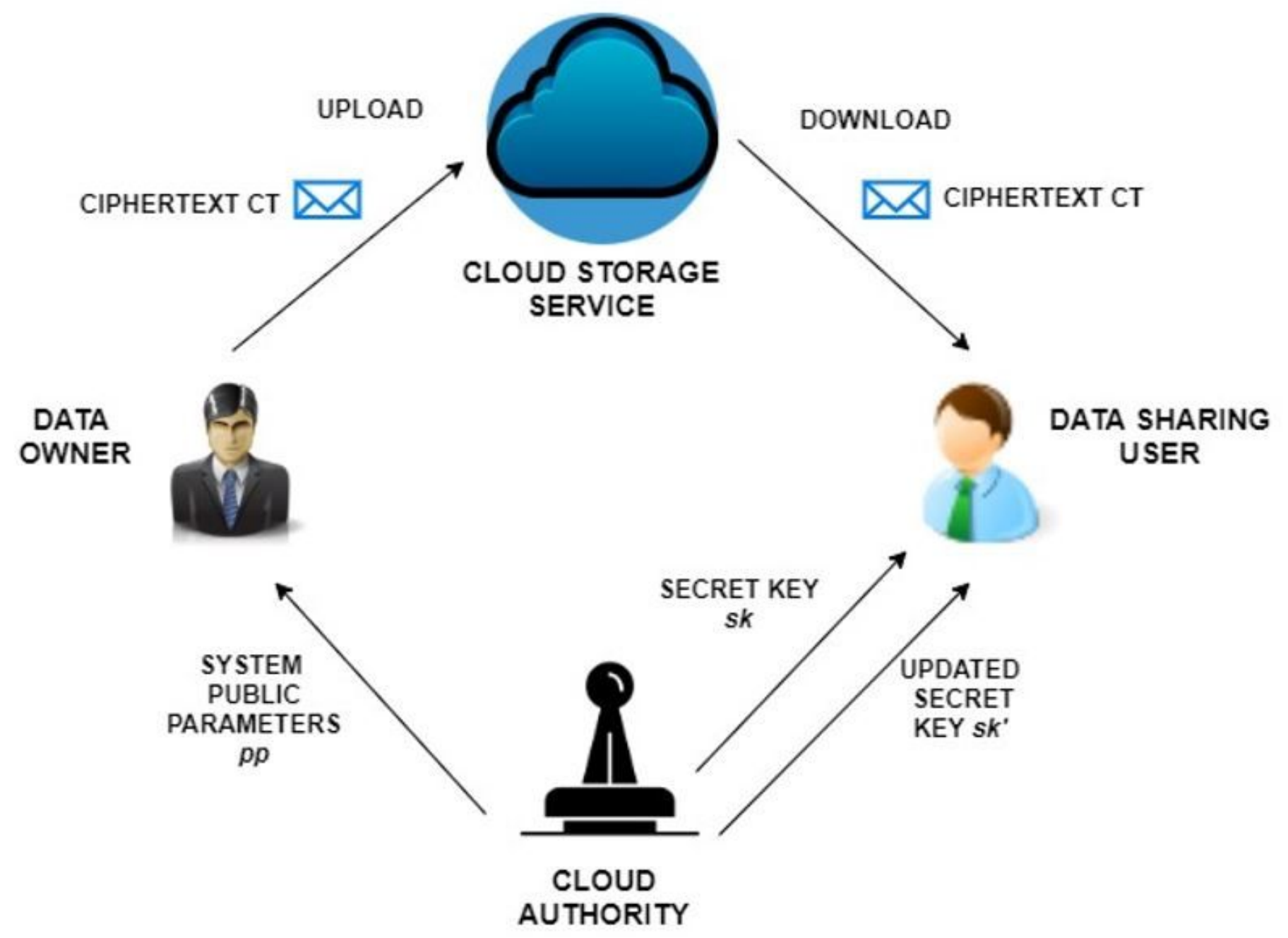

Figure 2

Attribute based encryption (ABE). 


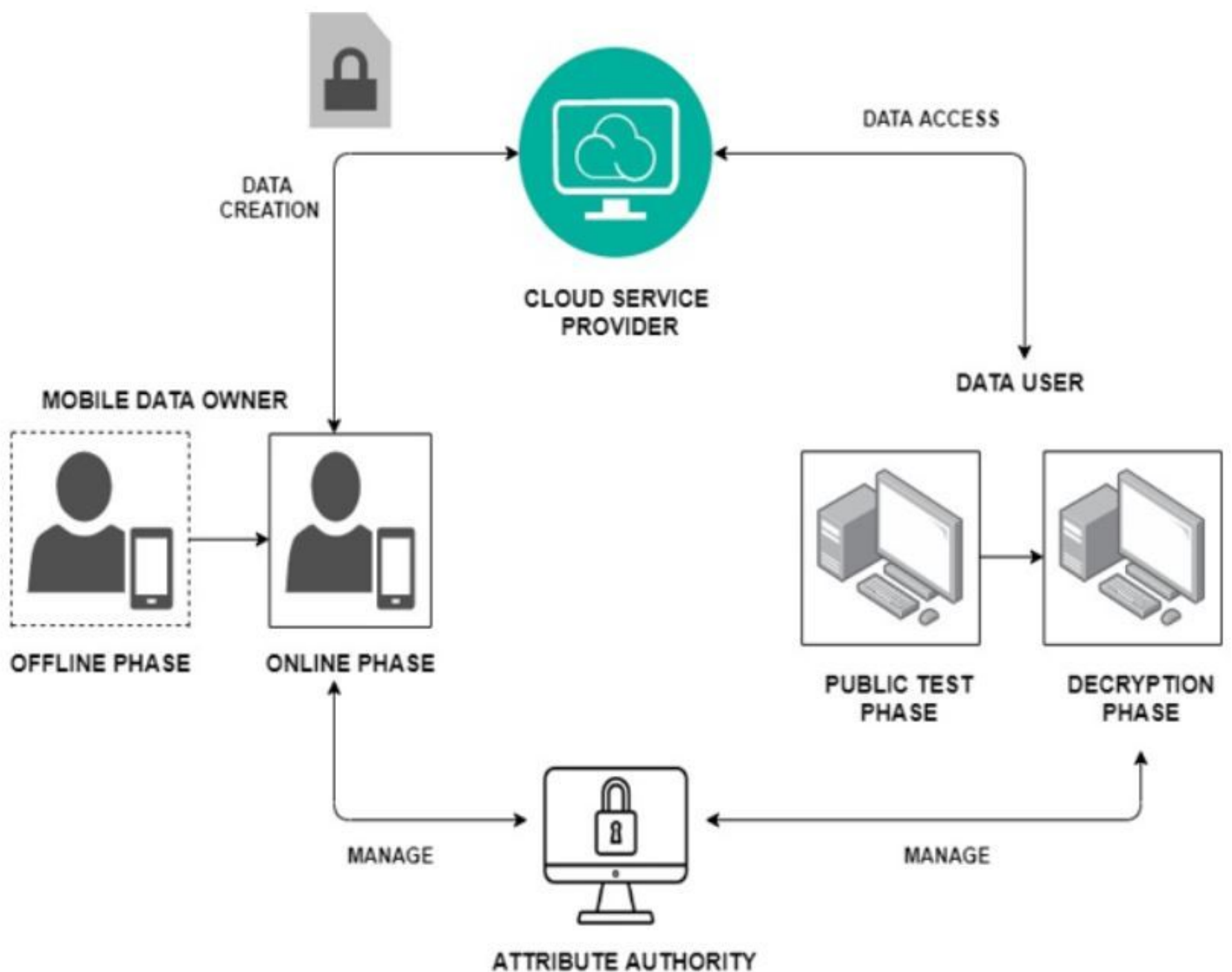

Figure 3

Attribute-based data sharing (ABDS) system model. 